\title{
The Early Stage of Cirrhotic Cardiomyopathy is a Risk Factor for Postreperfusion Syndrome During Liver Transplantation? A Retrospective Observational Study*
}

\author{
Ecaterina Scarlatescu ${ }^{1}$, Gabriela Droc ${ }^{2,3}$, Dana Tomescu ${ }^{1,2}$
}

Corresponding author:

Ecaterina Scarlatescu, M.D.

Department of Anaesthesia and Intensive

Care III, Fundeni Clinical Institute,

Sos Fundeni 258, Bucharest, Romania

E-mail: katyscarlatescu@yahoo.com

${ }^{*}$ An earlier version of the manuscript was presented as a poster at the $34^{\text {th }}$ Symposium on Intensive Care and Emergency Medicine in Brussels, in 2014.

\author{
'Department of Anaesthesia and Intensive Care III, Fundeni Clinical Institute, Bucharest, Romania \\ "University of Medicine and Pharmacy "Carol Davila" Bucharest, Romania \\ ${ }^{3}$ Department of Anaesthesia and Intensive Care I, Fundeni Clinical Institute, Bucharest, Romania
}

\begin{abstract}
Objectives: Despite normal or high cardiac output at rest, cirrhotic patients often exhibit abnormal cardiac function when subjected to stress. During the pretransplant cardiac workup, the diagnosis of cirrhotic cardiomyopathy would be easily missed without the aid of some supportive criteria because most patients have normal rest echocardiography. The period of highest hemodynamic instability during liver transplantation occurs after revascularization of the liver graft and is called postreperfusion syndrome. The aim of this study was to investigate if patients at risk for cirrhotic cardiomyopathy have a higher risk for postreperfusion syndrome during liver transplantation. The secondary purpose of the study was to assess if the supportive criteria used for cirrhotic cardiomyopathy evaluation correlate with the severity of the liver disease.
\end{abstract}

Material and Methods: The study group included 52 patients undergoing orthotopic liver transplantation for cirrhosis. The variables recorded were: demographic data, MELD and MELD Na scores, the length of the QT interval corrected for heart rate, plasma levels of brain natriuretic peptide (BNP), cold and warm ischemia times, vasopressor use, transfusional requirements and the occurrence of postreperfusion syndrome during transplantation.

Results: The patients were divided into 2 subgroups based on the occurrence of the postreperfusion syndrome. There was no statistically significant difference in BNP levels, length of QTC interval, MELD or MELD Na scores between the subgroups. BNP levels were correlated with QTC interval length ( $p=0.005)$, MELD and MELD Na scores $(p=0.025$ and $p=0.001$ ). The correlation between QTC interval length and the severity scores was not statistically significant.

Conclusions: Our study showed that postreperfusion syndrome was not correlated with the severity of the liver disease or with the presence of risk factors indicating cirrhotic cardiomyopathy. From the supportive criteria studied, only BNP levels correlate with the severity of the liver disease.

Key words: liver cirrhosis, liver transplant, cirrhotic cardiomyopathy 


\section{INTRODUCTION}

For a long period of time, patients with liver cirrhosis were considered having normal cardiac function, mainly because of the normal or supranormal cardiac output at rest. In 1953, Kowalski and Abelmann observed higher resting cardiac output and decreased systemic vascular resistance in patients with alcoholic cirrhosis, manifestations considered being the result of latent alcoholic cardiomyopathy (1). Only in the 1980s studies on nonalcoholic patients revealed the presence of a cardiac dysfunction in liver cirrhosis patients and the term cirrhotic cardiomyopathy (CCM) was used to describe it. Cirrhotic cardiomyopathy was defined by a working group in 2005 as a cardiac dysfunction in patients with liver cirrhosis characterized by an abnormal and blunted response to physiologic, pathologic, or pharmacologic stress, but normal to increased cardiac output and contractility at rest (2). The diagnostic criteria include: systolic dysfunction, diastolic dysfunction and supportive criteria which comprise: electrophysiological abnormalities, abnormal chronotropic response, prolonged QTc interval, enlarged left atrium, increased BNP and NT pro-BNP, increased troponin (3).

$56 \%$ of liver transplant patients in certain series manifested acute pulmonary edema during the early postoperative period, while $7-21 \%$ of early postoperative deaths were attributed to heart failure (4). Keeping in mind that liver transplant candidates are evaluated periodically by cardiologist, it means that cirrhotic cardiomyopathy cannot be easily detected by routine evaluation. Cirrhotic cardiomyopathy is a hidden clinical syndrome which becomes evident in some cases only when the patient is subjected to stress. The diagnostic of cirrhotic cardiomyopathy is difficult especially at early stages, because the clinical manifestations of systolic or diastolic dysfunction can be absent at rest, but one or more supportive criteria can be present.

Reperfusion of the liver graft is a critical period during liver transplantation procedures because hemodynamic instability can be profound at that moment. Even if it is usually of short duration, postreperfusion syndrome can have dramatic consequences. Postreperfusion syndrome (PRS) was defined by Aggarwal et al as persistent hypotension (a greater than 30\% drop below the anhepatic mean arterial blood pressure (MAP) within 5 minutes of reperfusion and sustained for at least 1 minute), which can be accompanied by asystole, arrhythmias and significant fibrinolysis (5). The clinical predictors of postreperfusion syndrome are not fully elucidated, nor the impact of postreperfusion syndrome on postoperative outcome $(6,7)$.

The primary aim of this study was to investigate whether if postreperfusion syndrome is more frequent in patients considered at risk for cirrhotic cardiomyopathy (with normal rest echocardiography, but with one or more supportive criteria present). A secondary objective was to assess whether if the diagnostic supportive criteria taken into account correlate with the severity of the liver disease evaluated with severity scores frequently used in clinical practice.

\section{MATERIALS AND METHODS}

After institutional review board approval (protocol number attributed by the Ethics Committee of Fundeni Clinical Institute 43073 in November 26, 2014), this retrospective observational descriptive study was conducted on 52 consecutive liver cirrhosis patients undergoing orthotopic liver transplantation in Fundeni Clinical Institute, Bucharest between January and November 2013. Exclusion criteria were: retransplantation, patients with known cardiac or renal disease, systolic or diastolic dysfunction on preoperative echocardiography at rest, the use of QT prolonging drugs and the recipients of organs from extended criteria donors. The following characteristics were used for the identification of extended criteria donors: age over 65 years, intensive care unit stay over 7 days, body mass index $>30$, biopsy proven steatosis $>40 \%$, peak serum natrium $>165 \mathrm{mmol} / \mathrm{l}$, alanine aminotransferase or aspartate aminotransferase $>3$ times normal value, serum total bilirubin $>$ $3 \mathrm{mg} / \mathrm{dl}$, positive serology for viral hepatitis, sepsis with positive blood culture, meningitis, history of extrahepatic malignancy and previous drug abuse (8).

The surgical technique was the same in all the cases: recipient hepatectomy performed with preservation of the inferior vena cava, triangulating cavocavostomy and caval cross- clamping without temporary porto-caval shunts. Anaesthesia was induced with propofol 1.5-2 $\mathrm{mg} / \mathrm{kg}$, fentanyl $2.5-3 \mathrm{mcg} / \mathrm{kg}$ and succinylcholine 1.5-2 $\mathrm{mcg} / \mathrm{kg}$ and was maintained with sevoflurane, fentanyl as needed (for Bispectral Index analysis of electroencephalogram value 40 to 60 ) and atracurium. Intraoperative monitoring consisted of: 2 lead electrocardiogram, invasive blood pressure and cardiac output measurement (PICCO plus continuous hemodynamic monitoring using a femoral arterial line), peripheral oxygen saturation, end-tidal carbon dioxide, diuresis, Bispectral Index and core temperature. During the 
preanhepatic phase, anesthetic management focused on maintaining an adequate cardiac preload, cardiac index and compensating blood loss. During both preanhepatic and anhepatic phases the calcium and potassium levels were carefully monitored and corrected. An ionized calcium level below $1 \mathrm{mmol} / \mathrm{l}$ was treated with calcium gluconate; hyperkalemia was treated with glucose and insulin after the acidosis correction. Vasopressor support (norepinephrine) was used (after the exclusion of low cardiac output) for a mean arterial pressure above $65 \mathrm{~mm} \mathrm{Hg}$ if hypotension persisted after hypovolemia correction. Before finishing hepatic veins anastomosis, the graft was perfused with $500 \mathrm{ml} 5 \%$ albumin through the portal vein. Reperfusion of the graft was achieved by simultaneous unclamping of the portal and caval veins after completion of portal and hepatic venous anastomoses.

The variables recorded were: demographic data, etiology of the liver disease, MELD and MELD Na scores, the length of the QT interval corrected for heart rate, plasma levels of brain natriuretic peptide (BNP), cold and warm ischemia times, vasopressor use, transfusional requirements and the occurrence of postreperfusion syndrome during liver transplant procedure. The QT interval was read from a preoperative standard 12 lead electrocardiogram and was corrected for the heart rate using Bazett's formula. For the identification of patients at risk for cirrhotic cardiomyopathy we used two of the supportive criteria from the recent definition of CCM: prolonged QT interval corrected for heart rate (over $440 \mathrm{msec}$ ) and increased BNP levels (cut off value $100 \mathrm{pg} / \mathrm{ml}$ ) (3). The criteria used for the diagnostic of the postreperfusion syndrome relied only on the hemodynamic changes that occurred at reperfusion of the graft. We defined postreperfusion syndrome as hypotension (mean arterial blood pressure $30 \%$ lower than the value immediately at the end of the anhepatic stage, lasting for more than 1 minute within 5 minutes after graft reperfusion), arrhythmias or asystole after unclamping (5).

Numerical data were tested for a normal distribution using the Shapiro Wilk's test. Numerical variables were presented as means and standard deviations and categorical data as numbers (percentages). The significance of differences between two subgroups of patients was tested using Mann-Whitney test. The correlation between continuous variables was assessed using bivariate correlations. For all statistical tests, significance was assumed at $p<0.05$. The statistical analysis was performed using SPSS Statistics v.19.1

\section{RESULTS}

The study group included 28 men (53.8\%) and 24 women. Mean $( \pm S D)$ age was $50.5( \pm 11.4)$. The etiology of the liver disease was: HVB+D cirrhosis $36.5 \%(n=19)$, HCV cirrhosis $25 \%(n=13)$, alcoholic cirrhosis $11.5 \%$ $(n=6), H C V$ cirrhosisthepatocarcinoma $11.5 \%(n=6)$, Wilson disease $7.6 \%(n=4)$ and cryptogenic cirrhosis $7.6 \%(n=4)$. In our study group $46.1 \%(n=24)$ of the patients had prolonged QTc ( longer than $440 \mathrm{msec}$ ).

In this study, the postreperfusion syndrome occurred in 33 patients (63.5\%). The patients were divided into 2 subgroups based on the occurrence of the postreperfusion syndrome. The patients with postreperfusion syndrome after unclamping were included in Group PRS+; the others (without postreperfusion syndrome) were included in Group PRS(table 1).

There was no statistically significant difference for demographic variables between the groups. The values for cold ischemia time (CIT), warm ischemia time (WIT), vasopressor requirements during preanhepatic and anhepatic phases, the number of units of red blood cell (RBC) and fresh frozen plasma (FFP) transfused were similar in both groups (table 1).

There was no statistically significant difference for BNP levels between the groups $(p=0.89)$. The length of the QTc interval was not significantly different between the groups. MELD and MELD Na scores had similar values in both groups (table 1). Bivariate correlations showed that BNP levels were correlated with QTc interval length $(p=0.005)$, MELD and MELD Na scores $(p=0.025$ and $p=0.001)$. The correlation between QTc interval length and the severity scores was not statistically significant ( $p=0.263$ for MELD and $p=0.187$ for MELD Na score).

\section{DISCUSSION}

In this study we found that: 1 . postreperfusion syndrome was not more frequent in patients at risk for cirrhotic cardiomyopathy (with normal preoperative echocardiography, but with at least one supportive criteria from the definition present: prolonged QTC interval or high BNP levels); 2. From the supportive criteria studied, only BNP levels correlated with the severity of the liver disease as assessed by MELD and MELD Na scores; 3. Higher BNP levels are associated with lengthening of the QTc interval in the cirrhotic patients in our study group.

The first conclusion of our study is interesting, because to our knowledge there had been no reports of 
Table 1 - Demographic and relevant data

\begin{tabular}{|c|c|c|c|c|}
\hline & $\begin{array}{l}\text { All patients } \\
(n=52)\end{array}$ & $\begin{array}{c}\text { Group PRS+ } \\
(n=33)\end{array}$ & $\begin{array}{c}\text { Group PRS- } \\
(n=19)\end{array}$ & P value* \\
\hline Age (years) & $50.5( \pm 11.4)$ & $50.54( \pm 10.97)$ & $50.94( \pm 12.41)$ & 0.904 \\
\hline Sex ratio M:F & $28: 24$ & $20: 13$ & $8: 11$ & \\
\hline $\mathrm{BNP}(\mathrm{pg} / \mathrm{ml})$ & $72.74( \pm 56.46)$ & $71.09( \pm 53.71)$ & $75.84( \pm 65.06)$ & 0.89 \\
\hline QTC (msec) & $455.53( \pm 39.24)$ & $459.62( \pm 37.35)$ & $447.62( \pm 42.86)$ & 0.134 \\
\hline MELD & $15.51( \pm 5.43)$ & $15.51( \pm 5.3)$ & $15.52( \pm 5.81)$ & 0.947 \\
\hline MELD Na & $18.9( \pm 6.22)$ & $18.66( \pm 5.98)$ & $19.52( \pm 6.76)$ & 0.746 \\
\hline CIT & $268( \pm 88.97)$ & $274.83( \pm 80.90)$ & $255.69( \pm 103.67)$ & 0.767 \\
\hline WIT & $29.51( \pm 19.63)$ & $28.55( \pm 16.78)$ & $31.25( \pm 24.48)$ & 0.962 \\
\hline Preanhepatic vasopressor(mcg/min) & $8.96( \pm 10.93)$ & $10.46( \pm 12.43)$ & $6.4( \pm 7.36)$ & 0.472 \\
\hline Anhepatic vasopressor $(\mathrm{mcg} / \mathrm{min})$ & $24.35( \pm 27.62)$ & $26.34( \pm 26.05)$ & $20.97( \pm 30.6)$ & 0.274 \\
\hline RBC units & $6.64( \pm 5.92)$ & $7.06( \pm 6.67)$ & $5.89( \pm 4.3)$ & 0.714 \\
\hline FFP units & $14.35( \pm 9.45)$ & $15.3( \pm 10.72)$ & $12.61( \pm 6.46)$ & 0.244 \\
\hline
\end{tabular}

Values are expressed as mean $\pm S D$. ${ }^{*}$ Data are analysed by Mann-Whitney $U$ test $\mathrm{RBC}=$ red blood cell; FFP $=$ fresh frozen plasma

the incidence and risk for postreperfusion syndrome in cirrhotic patients with normal echocardiography at rest and supportive criteria present. In a recent study by Zhen-Dong Xu et al (9) left ventricular diastolic dysfunction was a risk factor for postreperfusion syndrome.

Reportedly the incidence of PRS during OLT is quite different between studies. Paugam-Burtz et al (6) reported a PRS incidence of $25 \%$ in a retrospective study. In another study $55 \%$ of the patients had postreperfusion syndrome (7). In a smaller study, Bezinover et al (10) reported PRS in 16 patients from a group of 17 patients. In our study we had a high incidence of PRS - $63.5 \%$. This is an interesting finding because we defined PRS only on hemodynamic criteria, not taking into account hyperfibrinolysis, leading to a possible underestimation of the patients with PRS.

In our study, BNP levels correlated with MELD and MELD Na scores, which agrees with other data published. In a study by Saner et al (11) BNP levels also correlated with MELD score, but also with diastolic dysfunction in cirrhotic patients. According to the authors of the study, BNP could be used as a screening tool for cirrhotic cardiomyopathy in patients with liver cirrhosis. In our study, BNP levels are correlated with the severity of the liver disease in patients with normal echocardiography. This result can be explained by the fact that the diagnosis of diastolic dysfunction in cardiac pretransplant evaluation was based on transmitral Doppler flow velocity, without the use of tissue Doppler, which is a limitation of our study.

The etiology and risk factors for postreperfusion syndrome are still the subject of controversies. Multiple factors regarding the recipient, the surgery and the graft can have an impact on the incidence and severity of the postreperfusion syndrome. Knowledge of recipient-dependent risk factors is extremely important. Preoperative identification of recipients at high risk for postreperfusion syndrome would allow planning a surgical technique and a graft matching in order to minimize the occurrence and/or the severity of hemodynamic instability after unclamping. Our study indicated that the presence of supportive criteria for cirrhotic cardiomyopathy in patients with normal rest echocardiography is not a risk factor for postreperfusion syndrome during liver transplantation.

\section{CONCLUSIONS}

The presence of the supportive criteria for cirrhotic cardiomyopathy with normal echocardiography may be considered an early stage of cardiac involvement in cirrhotic patients which is not a risk factor for postreperfusion syndrome during liver transplant surgery. The supportive criteria like length of QTc interval or BNP levels can be used as periodic screening tools in cirrhotic patients listed for transplantation, unmasking the early beginning of cirrhotic cardiomyopathy.

\section{Author's contribution}

All authors contributed to the design, drafting and final approval/preparation of the article. 


\section{Conflicts of interest}

None to declare

\section{Funding}

The authors have no funding to declare.

\section{REFERENCES}

1. Kowalski HJ, Abelmann WH. The cardiac output at rest in Laennec cirrhosis. J Clin Invest. 1953 Oct;32(10):1025-33.

2. Zardi EM, Abbate A, Zardi DM, Dobrina A, Margiotta D, Van Tassell BW, et al. Cirrhotic cardiomyopathy. J Am Coll Cardiol. 2010 Aug 10;56(7):539-49.

3. Møller S, Henriksen JH. Cardiovascular complications of cirrhosis. Gut. 2008 Feb;57(2):268-78.

4. Therapondos G, Flapan AD, Plevris JN, Hayes PC. Cardiac morbidity and mortality related to orthotopic liver transplantation. Liver Transpl. 2004 Dec;10(12):1441-53.

5. Aggarwal S, Kang Y, Freeman JA, Fortunato FL, Pinsky MR. Postreperfusion syndrome: cardiovascular collapse following hepatic reperfusion during liver transplantation. Transplant Proc. 1987 Aug; 19(4 Suppl 3):54-5.

6. Paugam-Burtz C, Kavafyan J, Merckx P, Dahmani S, Sommacale D, Ramsay M, et al. Postreperfusion syndrome during liver transplantation for cirrhosis: outcome and predictors. Liver Transpl. 2009 May; 15(5):522-9.

7. Hilmi I, Horton CN, Planinsic RM, Sakai T, Nicolau-Raducu R, Damian $\mathrm{D}$, et al. The impact of postreperfusion syndrome on short-term patient and liver allograft outcome in patients undergoing orthotopic liver transplantation. Liver Transpl. 2008 Apr;14(4):504-8.

8. Fischer-Fröhlich CL, Lauchart W. Expanded criteria liver donors (ECD): effect of cumulative risks. Ann Transplant. 2006;11(3):38-42.

9. Xu ZD, Xu HT, Yuan HB, Zhang H, Ji RH, Zou Z, et al Postreperfusion syndrome during orthotopic liver transplantation: a single-center experience. Hepatobiliary Pancreat Dis Int. 2012 Feb;11(1):34-9.

10. Bezinover D, Kadry Z, McCullough P, McQuillan PM, Uemura T, Welker K, et al. Release of cytokines and hemodynamic instability during the reperfusion of a liver graft. Liver Transpl. 2011 Mar; 17(3):324-30.

11. Saner FH, Neumann T, Canbay A, Treckmann JW, Hartmann M, Goerlinger $\mathrm{K}$, et al High brain-natriuretic peptide level predicts cirrhotic cardiomyopathy in liver transplant patients. Transpl Int. 2011 May; 24(5):425-32. 